\title{
NOISE-ENHANCED HETERODYNING
}

\author{
M I Dykman
}

Department of Physics, Stanford University, Stanford, CA 94305, USA.

D G Luchinsky', P V E McClintock, N D Stein and N G Stocks ${ }^{2}$

School of Physics and Materials, Lancaster University, Lancaster, LA1 4YB, UK.

\section{ABSTRACT}

A new form of heterodyning is reported, related to stochastic resonance, in which a heterodyne signal can be enhanced by adding noise.

One of the important physical problems of information processing and transfer is how to control the signal-to-noise ratio (SNR). Usually, this ratio decreases with increasing intensity of noise. However, under certain circumstances it behaves in the opposite way. The phenomenon of the noise-induced increase of SNR was called stochastic resonance (SR) [1]. It has attracted much attention recently (see [2]). Most of the data on SR has been obtained for bistable systems driven by noise and by a low-frequency periodic force. The onset of $\mathrm{SR}$ in these systems is related to the fact that the probabilities $W_{n m}$ of transitions between co-existing stable states $(n, m=1,2)$ increase exponentially, in the case of Gaussian noise, with increasing noise intensity $D$. A low-frequency external periodic force $A_{0} \cos \Omega t$ modulates the activation energies and as a result the transition probabilities $W_{n m}$ are modulated too. In turn, the modulation of $W_{n m}$ gives rise to a modulation of the populations of the stable states. For a symmetrical double-well potential, the force periodically makes one of the wells deeper than the other, and the system occupies it with a larger probability. As a result, the amplitude of the oscillations is proportional to the relatively large difference $x_{1}-x_{2}$ in the values of the coordinate in the stable states $x_{n}$.

When the above mechanism comes into play through the onset of fluctuational transitions, the amplitude of the periodic signal increases with increasing noise intensity, in a certain range of $D$, and the SNR increases with $D$, too. It works, provided (i) the stationary populations of the states in the absence of the periodic force are nearly equal to each other [3], and (ii) the frequency of the force is much smaller than the reciprocal relaxation time $t_{r}^{-1}$ of the system, so that the transitions are likely to occur within the period $2 \pi / \Omega$.

The frequency-selective response of bistable systems, and also the fact that the SNR increases with increasing noise intensity, makes it interesting to apply the idea of SR to heterodyning so as to obtain a form of the phenomenon that is enhanced rather than suppressed by noise. In heterodyning, two high-frequency fields, one of them a signal and the other a reference field, are mixed nonlinearly to generate a signal at a difference frequency. In this paper we report and discuss

${ }^{1}$ Permanent address: VNIIMS, Andreevskaya nab. 2, Moscow, 117965, Russia.

${ }^{2}$ Present address: Dept. of Engineering, University of Warwick, Coventry, CV4 7AL, UK.

(c) 1993 American Institute of Physics 
a new form of the phenomenon, noise-enhanced heterodyning (NEH), that occurs in bistable systems and is highly frequency selective. We have investigated it theoretically and by analogue electronic simulation.

We shall illustrate the effect on a model of an overdamped bistable system driven by three time-dependent forces representing respectively the reference and input signals, and the noise. The motion of the system is described by the equation

$$
\frac{d x}{d t}=-U^{\prime}(x)+A_{\mathrm{ref}} x \cos \omega_{0} t+A_{\text {in }}(t) \cos \left[\omega_{0} t+\phi(t)\right]+f(t)
$$

Here, the term $\propto A_{\text {ref }}$ is the reference signal of a given frequency $\omega_{0}$ (the corresponding force is applied multiplicatively), and the term $\propto A_{\text {in }}(t)$ is the modulated high-frequency input signal (applied additively). The functions $A_{\text {in }}(t)$ and $\phi(t)$ are slowly varying as compared with $\cos \omega_{0} t$, and it is their variation in time that has to be revealed via heterodyning. The heterodyning can be characterized by the low-frequency signal at the output, $x(t)$, for $A_{\text {in }}=$ const and $\phi=\Omega t+$ const, with $\Omega \ll \omega_{0}$, i.e., for a monochromatic input signal with the frequency $\omega_{0}+\Omega$ slightly different from the frequency $\omega_{0}$.

We shall assume that the double-well potential of the system $U(x)$ has equallydeep wells, corresponding to standard SR, and is of the form

$$
U(x)=-\frac{1}{2} x^{2}+\frac{1}{4} x^{4}
$$

The minima of the potential (2) (the stable states of the system) lie at $x_{n}=$ $(-1)^{n}, n=1,2$, and the characteristic (dimensionless) relaxation time of the system $t_{r} \equiv 1 / U^{\prime \prime}\left(x_{n}\right)=1 / 2$. The analysis of heterodyning in bistable systems is not limited to the particular form of Eqs.(1),(2). However, the explicit expressions take on a simple form for this model. They are further simplified in the case where the frequencies of the input and reference signals are high compared with the reciprocal relaxation time of the system, $\omega_{0} \gg t_{r}^{-1}$.

The term $f(t)$ in (1) is a zero-mean Gaussian noise. In view of the possible applications we will allow for noise that consists of two independent components, of low and high frequency respectively, with the latter being randomly modulated vibrations at frequency $\omega_{0}$ (which might result from the scattering of the signal at frequency $\omega_{0}$ ):

$$
f(t)=f_{l f}(t)+f_{h f}(t), \quad f_{h f}(t)=\operatorname{Re}\left(\tilde{f}_{h f}(t) \exp \left(-i \omega_{0} t\right)\right),
$$

The power spectrum of the low-frequency noise $f_{l f}(t)$ is assumed to be flat up to $\omega \sim \omega_{c} \gg t_{r}^{-1}\left(\omega_{c}\right.$ may be small compared to $\left.\omega_{0}\right)$.

For $\omega_{0} \gg t_{r}^{-1}$ the motion of the system consists of fast oscillations at frequency $\omega_{0}$ (and its overtones) superimposed on a slower motion. To first order in $\omega_{0}^{-1}$ the equation for the smooth part of the coordinate, $x^{(s m)}$, is of the form

$$
\dot{x}^{(s m)}=-U^{\prime}\left(x^{(s m)}\right)+A(t) \sin \phi(t)+f^{(0)}(t), \quad A(t)=\frac{A_{\text {ref }}}{2 \omega_{0}} A_{\text {in }}(t)
$$


where $f^{(0)}(t)=f_{l f}(t)-\left(A_{\text {ref }} / 2 \omega_{0}\right) \operatorname{Im} \tilde{f}_{h f}(t)$ is the noise which, in view of the above comments, will be assumed white (its correlation time $\sim \omega_{c}^{-1} \ll$

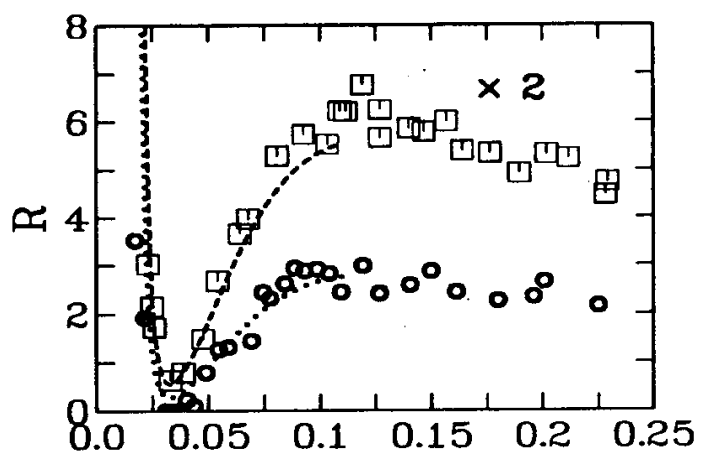

D

Fig.1 NEH for white (circles) and highfrequency (boxes) noise. The dashed and dotted lines are theory. $t_{r}$ ). The noise intensity $D$ is composed of a weighted sum of low and high frequency contributions.

The dynamics of the system described by Eqs.(2), (4), for $A(t), \phi(t)$ varying slowly over the time $t_{r}$, has been investigated in detail in the context of stochastic resonance. For sufficiently small $A$ the general analysis of the response can be done in terms of linear-response theory [3]. In the particular case of a monochromatic force of frequency $\Omega$, i.e., $A(t) \sin \phi(t)=A \sin \Omega t$, a $\delta$-shaped spike arises in the power spectrum of the coordinate $x^{(s m)}$ at $\omega=\Omega$ on top of the broad spectrum.

As in standard stochastic resonance, NEH can be characterized by the ratio $R$ of the intensity (area) of this spike (which is just proportional to the squared amplitude of forced vibrations at frequency $\Omega$ ) to the value of the power spectrum at the same frequency for $A=0$

$$
R=\pi \frac{A^{2}\left(x_{2}-x_{1}\right)^{2}}{16 D^{2}} \frac{W^{2}+\Omega^{2} t_{r}^{2} \tilde{D}^{2}}{W+\Omega^{2} t_{r}^{2} \tilde{D}}, \quad \tilde{D}=4 D /\left(x_{2}-x_{1}\right)^{-2} .
$$

Eq.(5) was shown in [4] to hold for arbitrary $\Omega / W(W \propto \exp (-\Delta U / D)$ is the probability of the interwell transitions) to lowest order in $\Omega t_{r}, \tilde{D} t_{r}, W / \tilde{D} \ll 1$. It follows from (5) (and also from the more general expression for the SNR) that, in the range of the noise intensities where $\Omega \tilde{D} t_{r} \lesssim W \lesssim t_{r}^{-1}$, the SNR increases with increasing noise intensity. The fact that this increase is quite sharp - nearly exponential - means that noise-enhanced heterodyning would be expected to arise in a bistable system, whether driven by a low- or a high-frequency noise (or both).

The onset of NEH has been investigated experimentally by means of ana$\log$ electronic simulation. The design will be discussed elsewhere. In Fig. 1 the experimentally measured values of the heterodyne SNR are compared with the theoretical predictions for low- and high-frequency noises. It is clearly seen that, in both cases, there is an interval of noise intensities where the SNR sharply increases with $D$. Below and above this interval the SNR decreases with increasing noise intensity. The experimental data are in satisfactory agreement with the linear-response theory; we note that the latter does not contain any adjustable parameters (we have used an expression [4] that allows for the corrections $\sim D / \Delta U$ 
omitted in (5)). It has been shown experimentally that $R$ is proportional to the squared amplitudes of the reference and input signals, and also that $R \propto \omega_{0}^{-2}$ over a broad range of $\omega_{0}$ up to $\omega_{0} \approx$ $2 t_{r}^{-1}$ as shown in Fig.2.

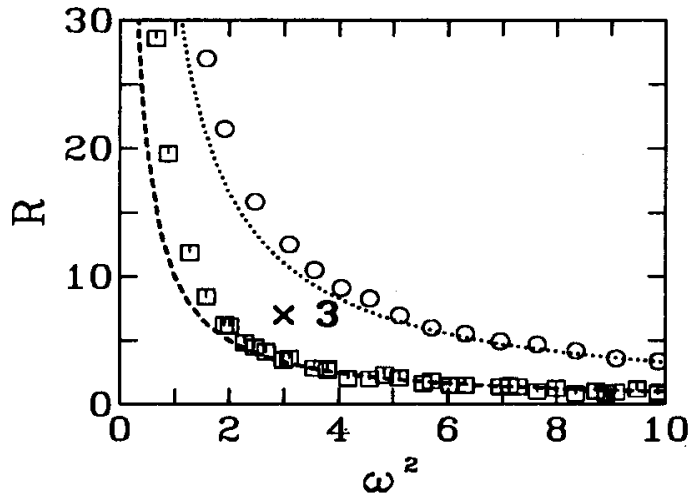

Fig.2 NEH dependence on the squared frequency of the reference signal for $\Omega=0.0031$ with noise intensities $\mathrm{D}=0.015$ (circles) and $\mathrm{D}=0.14$ (boxes). The dashed and dotted lines $\propto 1 / \omega_{0}^{2}$.

Of particular interest is the dependence of the SNR on the modulation frequency $\Omega$. It follows from (5) that in the interesting range where $R$ sharply increases with noise intensity, i.e. in the range $D \ll \Delta U, R$ also increases quite sharply with $\Omega$, from the value that corresponds to the SNR in the neglect of the intrawell motion, $R=R_{0} \approx$ $\pi A^{2} W / 4 D \tilde{D}$ for very small $\Omega$, up to the value that corresponds to the SNR in the neglect of the interwell transitions, $R \approx \pi A^{2} / 4 D$. As in the case of SR [2], a simple way to avoid frequency dispersion is to apply two-state filtering where the quantities of interest are the values of the coordinate coarse-grained over the vicinities of the stable states. For $\Omega \ll t_{r}^{-1}$ the value of the SNR at the output of a two-state filter is given approximately by $R_{0}$.

In conclusion, we have demonstrated, theoretically and experimentally, that bistable systems can be used to obtain heterodyning in which not only the amplitude of the signal at the output, but also the signal-to-noise ratio increases with increasing intensity of the noise.

The work was supported by the Science and Engineering Research Council (UK), by the EC, by the Royal Society of London, and by the Gosstandart of Russia.

\section{REFERENCES}

1. R. Benzi, A. Sutera and A. Vulpiani, J. Phys. A 14, L453 (1981); C. Nicolis, Tellus 34, 1 (1982); R. Benzi, G. Parisi, A. Sutera and A. Vulpiani, Tellus 34, 10 (1982).

2. Special issue of J. Stat. Phys. 70, no. $1 / 2$ (1993).

3. M.I. Dykman, R. Mannella, P.V.E. McClintock, and N.G. Stocks, JETP Lett. 52, 141 (1990).

4. M.I. Dykman, R. Mannella, P.V.E. McClintock, and N.G. Stocks, Phys. Rev. Lett. 68, 2985 (1992). 